\title{
Daily-life monitoring of stroke survivors motor performance: the INTERACTION sensing system
}

\author{
A. Tognetti ${ }^{1,2}$, F. Lorussi ${ }^{1}$, N. Carbonaro ${ }^{1}$, D. De Rossi ${ }^{1,2}$, G. De Toma ${ }^{3}$ \\ C. Mancuso ${ }^{3}$, R. Paradiso ${ }^{3}$, H. Luinge ${ }^{4}$, J. Reenalda ${ }^{5}$, E. Droog ${ }^{6}$, P.H. Veltink ${ }^{6}$
}

\begin{abstract}
The objective of the INTERACTION Eu project is to develop and validate an unobtrusive and modular system for monitoring daily life activities, physical interactions with the environment and for training upper and lower extremity motor function in stroke subjects. This paper describes the development and preliminary testing of the project sensing platform made of sensing shirt, trousers, gloves and shoes. Modular prototypes were designed and built considering the minimal set of inertial, force and textile sensors that may enable an efficient monitoring of stroke patients. The single sensing elements are described and the results of their preliminary lab-level testing are reported.
\end{abstract}

\section{INTRODUCTION}

Stroke survivors are trained to recover adequate control over their movements to optimise daily-life functional performances. Continuous daily-life monitoring of the functional activities of stroke patients in their physical interaction with the environment is essential for optimal guidance of rehabilitation therapy by medical professionals and coaching of the patient. Such performance information cannot be easily obtained in daily life conditions with current monitoring systems.

The objective of the INTERACTION project [1] is to develop and validate an unobtrusive and modular sensing system for monitoring daily life activities of stroke subjects, evaluating their physical interactions with the environment and training motor function of upper and lower extremities. The system is unobtrusively integrated in clothing (e-textile) and includes fabric-based and distributed inertial sensing, providing tele-monitoring and adaptive on-body feedback capabilities [2].

This work describes the INTERACTION sensing platform developed in the first two years of the project. From a technical point of view, the main objective of the project is to exploit smart sensory fusion of textile, flexible and inertial sensors in order to develop an effective and as unobtrusive as possible wearable sensing system. Through this prototype it will be possible to provide full body motion monitoring (upper and lower arms including hands and feet, trunk, center of mass) and to detect the patient kinetic interaction with the environment at the body extremities (sensing gloves

\footnotetext{
${ }^{1}$ Research Center E.Piaggio, University of Pisa, Italy a.tognetti at centropiaggio.unipi.it

${ }^{2}$ Information Engineering Department, University of Pisa, Italy

${ }^{3}$ Smartex S.r.l., Pisa, Italy

${ }^{4}$ Xsens Technologies, Enschede, the Netherlands

${ }^{5}$ Roessingh Research and Development, Enschede, the Netherlands

${ }^{6}$ Biomedical Signals and Systems, University of Twente, Enschede, the Netherlands
}

and shoes). In particular, a set of wireless on-body devices fully integrated in a modular textile platform (shirt, trousers, gloves, shoes) were developed and are described in the current paper. In addition, preliminary lab level tests on the single sensing elements are shown and discussed.

\section{MATERIALS AND METHODS}

\section{A. Wearable sensors}

The INTERACTION modular system was developed starting from a set of wearable textile and non-textile sensors available from the partner consortium: inertial measurement units (IMUs), knitted piezoresistive fabrics (KPFs), textile electrodes and force sensitive resistors (FSRs).

A minimum set of IMUs (MTw by XSENS [3]) was applied to track the orientation of body segments as described by Roetenberg in [4].

The KPFs were used both in single layer configuration, hereinafter called strain sensors, to measure fabric deformations due to joint movements as reported in [5] and as double layer sensors, hereinafter referred to as goniometers, to measure angle between body segments as described in [6], [7].

The textile electrodes were implemented using woven conductive fibers, elastic fibers and natural or synthetic yarns as reported by Caldani in [8]. Textile electrodes were specifically designed for EMG acquisition with a square shape (10 mm side) and inter-electrode distances of $20 \mathrm{~mm}$.

The FSR sensors were employed to measure patient force interaction with the environment in upper and lower extremities.

\section{B. Requirements analysis and system design}

The daily life monitoring system was designed starting from user requirements derived from questionnaires sent to stroke professionals and interviews held with selected stroke patients. The project required functionalities mainly consisted in: 1) reaching distance and frequency and type of grasp of the affected and unaffected arms; 2) EMG for relating shoulder abductor effort to elbow extension and evaluating possible pathological synergies; 3 ) weight distribution and kinematic asymmetries between affected and unaffected legs in gait/ambulation. User requirements and technical state of the art (i.e. including the available wearable sensors described above) were translated into functional requirements and technical specifications. This design phase was aimed at reducing the overall system complexity and increasing the usability and comfort for the user, with particular focus on 

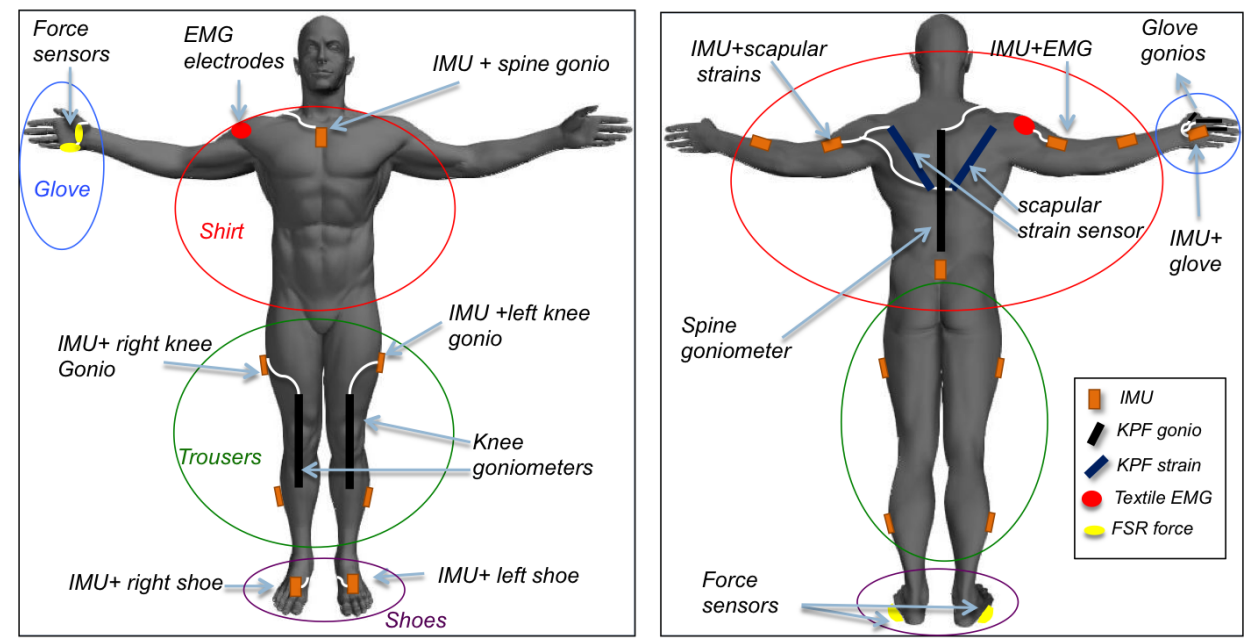

Fig. 1. Textile-integrated sensing system for daily-life assessment of motor performance in stroke (i.e. right side affected), including inertial sensor modules on main body segments, shoulder abductor EMG, shoulder strain sensing, spine and hand goniometers, force sensing under shoes and in glove. The system is divided into shirt, trousers, shoes and gloves. Patient specific combinations of modules can be chosen.

reducing the time to wear the system and the calibration effort. The derived specifications of the INTERACTION sensing system are illustrated in Fig. 1. The system is modular and divided into shirt, trousers, shoes and gloves. Different combination can be chosen to address specific patient needs.

\section{Modular prototypes}

The shirt module was designed to continuously track (i) elbow flexion/extension, (ii) position of hands relative to elbow, shoulder and sternum and (iii) compensatory trunk movements. Elbow flexion/extension and hand position relative to elbow and shoulder will be estimated from orientation of upper arm and forearm, derived from IMUs on both arm segments as described in [9]. Shoulder to sternum position is more difficult to estimate, since the shoulder is a complex deformable system. For this reason, KPF strain sensors were placed in correspondence of the scapulothoracic joint to detect scapular sliding and estimate the position of the shoulder relative to the spine and to the sternum. Trunk flexion is estimated by combining the information of the sternum and sacrum IMUs with the KPF goniometer along the spine as described in [10]. EMG of the deltoid muscle at the affected side was included to assess the activation of the shoulder abductor to oppose arm loading, which may be pathologically coupled to elbow flexion [11].

The kinaestetic and kinetic glove module was designed to identify reaching and grasping activities in their frequency and duration. The type of grasp will be assessed using KPF goniometer instrumented gloves, specifically designed for the project and with the distal part of the fingers left uncovered in order to preserve touch sensation. Three KPF goniometers were integrated in the metacarpo-phalangeal area of thumb, index and medium fingers. This configuration was considered sufficient to recognise a minimal set of functional hand grips. The global orientation and movement of the hand will be assessed using the IMU on the dorsal side of the hand. FSR sensors were also integrated in the palm and lateral side of the glove to measure contact pressures and give an adequate indication of hand loading in stroke patients, in order to evaluate the use of upper extremities to support body weight (e.g. during standing up tasks).

Sensing trousers were conceived to measure sitting, standing and walking activities that can be detected using IMUs on the thighs and shanks [12]. The knee KPF goniometers were also included to improve the quality of the movement detected through data fusion with IMU measurements.

The shoe modules were designed to estimate body center of mass (CoM) movement relative to center of pressure during daily-life gait, following the approach described by Schepers in [13]. In order to develop unobtrusive instrumented shoes, we chose 1D force sensing at least under the heel and forefoot and only one inertial sensor module connected to the dorsal side of the foot. Continuous estimation

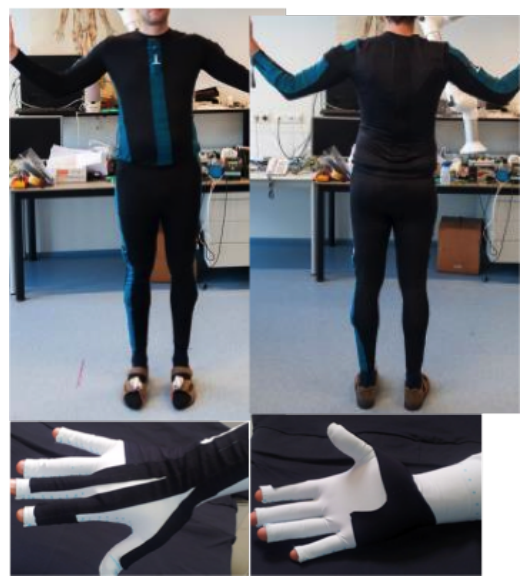

Fig. 2. Modular and wireless INTERACTION textile-integrated sensing system. Upper figures: shirt, trousers and shoes. Lower figures: kinaestetic and kinetic glove

of relative foot position is essential in this approach. It will 


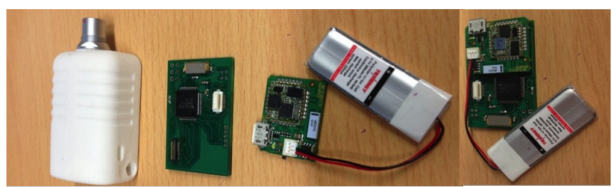

Fig. 3. From left to right: goniometer box, goniometer board, Wireless IMU + battery, IMU attached to goniometer board.

be derived from estimating the orientations of leg segments and pelvis using IMUs on thighs, shanks and sacrum. In addition, the sensing shoes will derive kinematic, kinetic and temporal parameters of patient gait. This includes estimation of walking kinematic, kinetic and asymmetries in gait.

The modular textile platform is shown in Fig. 2 and it includes the garments, the textile sensors, the interconnections, the connectors and the IMU pockets. In order to provide a high level of integration and miniaturisation, to reduce the number of electronic boxes on the garments and to enable wireless streaming of acquired data, textile sensor acquisition electronics were connected to IMU boards, exploiting an available digital channel, and integrated in an unique box specifically designed for our application (as shown in Fig. 3 for a KPF goniometer).

\section{RESULTS}

This section reports preliminary results obtained by the wearable system. These tests were aimed at assessing the validity of the single sensing components at the lab level, with particular focus on textile sensors validation (i.e. IMU performance has been widely validated in literature) and on sensor fusion between textile sensors and IMUs. More deep analysis in terms of motor performance reconstruction will be investigated in future work (i.e. test on human subjects simulating daily-life conditions and on patients both in clinical and in daily life will be performed in the final year of the project).

\section{A. Textile electrodes for $E M G$}

EMG of the deltoid muscle measured by the textile electrodes was compared with the output of a commercial

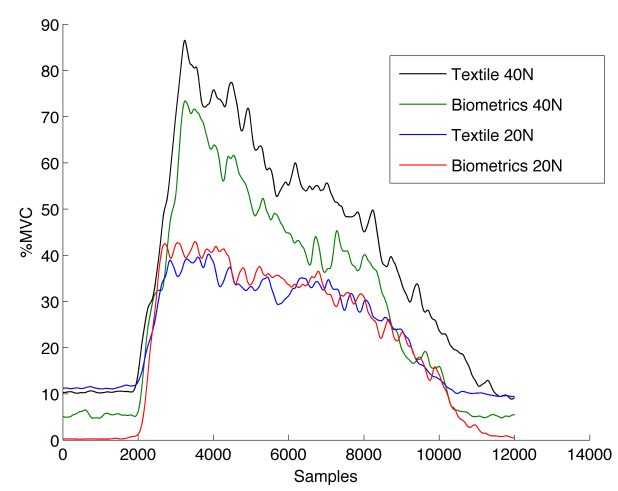

Fig. 4. Rectified EMG (\% of Maximum Voluntary Contraction) comparison between textile and commercial electrodes acquired in $20 \mathrm{~N}$ and $40 \mathrm{~N}$ load conditions (signal sampled at $2 \mathrm{~K} \mathrm{Sa} / \mathrm{s}$ )

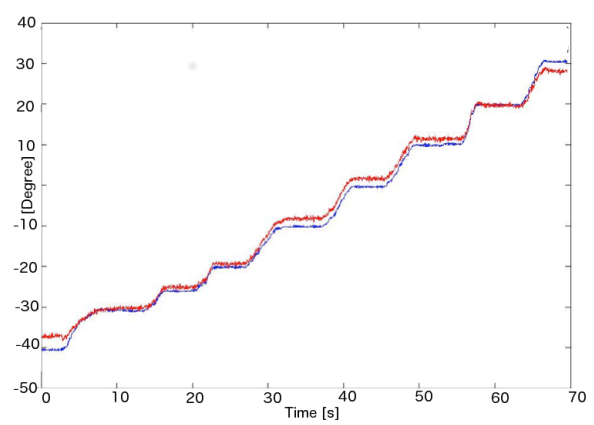

Fig. 5. Trend of the forefinger goniometer of the glove (red line) in comparison with Biometrics electrogoniometer (blue line) in a sample dynamic test

EMG system for different loading conditions. As qualitatively shown in Fig. 4, textile electrodes give comparable measurements with respect of commercial electrodes.

\section{B. KPF goniometers}

KPF strain and angular sensors were bench tested in quasi static conditions using dedicated instrumentation. KPF goniometers static characterisation pointed out a good sensitivity (in the order of $1000 \Omega /$ Degree) with acceptable calibration error (in the order of 5 Degrees). Dynamic characterisation of KPF goniometers was performed on the garments in comparison with standard instrumentation (elctrogoniometers by Biometrics [14]). Results, shown in Fig. 5 for a forefinger flexion test using the sensing glove, were very promising and confirmed quasi-static accuracy. Comparable results were obtained with knee and back goniometers.

\section{Force Sensors in glove and shoes}

Five FSR sensors (two in the palm of the hand close to the heel and two in the dorsal side) were integrated in the glove to give a qualitative indication of hand loading in stroke patients. The resultant force in the two sensing areas (yellow circles on the right hand in Fig. 1) was calculated and the resulting signals were quantised in three levels through an adaptive threshold algorithm: no contact, slight contact, high contact. The high contact condition may mean that the patient

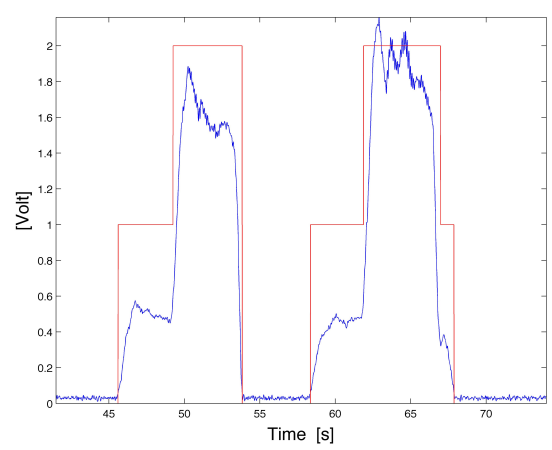

Fig. 6. Signals deriving from the force sensors placed on the heel of the hand, with the hand in free position, laying on a surface and exerting forces during a standing up phase. Blue line is the raw force signal while red line represents the discretized signal which codes the activity. 

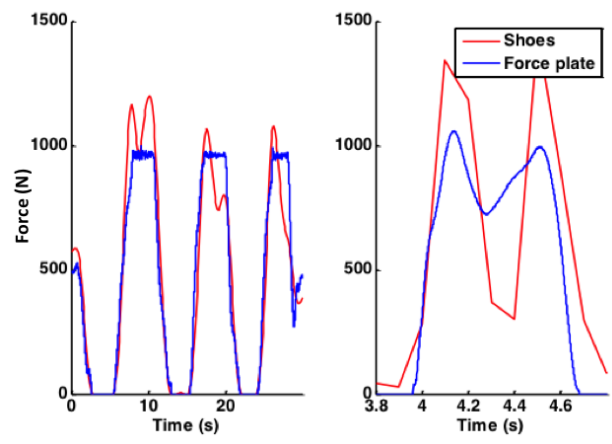

Fig. 7. Ground reaction force estimation based on two FSR sensors (red line) in the shoe compared to a force plate measurement (blue line). On the left: low-pass filtered ground reaction force for use in estimating body of mass movement (cut-off frequency of $0.4 \mathrm{~Hz}$ ); On the right: ground reaction force during a stance phase of continuous gait.

is using his hand to support his weight during certain motor tasks (e.g. standing up from a seated position). Fig. 6 shows the glove signals while the subject was using the hand to support his weight during a standing-up task.

Two FSR sensors were integrated in each shoe to estimate the ground reaction force and derivate the body center of pressure. Ground reaction estimation was compared with a force plate measurement as shown in Fig. 7.

\section{Sensor Fusion}

Sensor fusion was tested on knee movements by merging data deriving from IMUs placed on the thigh and on the calf and the goniometers placed on the femoral-tibial joints following the technique described in [15]. The considered movement is the flexion and extension of the knee. Data were acquired on different motor tasks, such flexion extension in monopodalic contralateral standing position at different velocities. A first graphical evaluation of the system performances is given in Fig. 8.

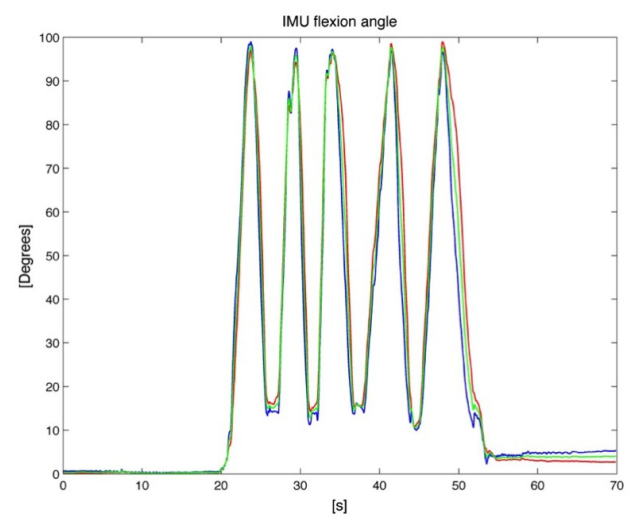

Fig. 8. Sensor fusion in a knee flexion-extension experiment. Blue line represents the goniometer angle trend, while the red one is the angle extracted from the two IMUs. Fused data are represented by the green graph.

\section{CONCLUSIONS}

This paper describes the development and preliminary testing of a modular sensing platform for the remote monitoring of the functional activities of stroke patients in their physical interaction with the environment. Sensing system design and development are discussed. Preliminary lab-level results of tests performed on the single sensing elements are also reported. Future activities will be aimed at validating the whole system on stroke patients through clinical trials.

\section{ACKNOWLEDGMENT}

This research has been supported by the EU 7th framework project INTERACTION (FP7-ICT-2011-7-287351).

\section{REFERENCES}

[1] "http://cms.interaction4stroke.eu/drupal/."

[2] e. a. Veltink, P H, "Interaction, training and monitoring of daily-life physical interaction with the environment after stroke," in Proceedings of XII International Symposium on 3D Analysis of Human Movement, Bologna, Italy, 2008.

[3] "www.xsens.com/products/mtw-development-kit-lite/mtw/."

[4] D. Roetenberg, H. J. Luinge, C. T. Baten, and P. H. Veltink, "Compensation of magnetic disturbances improves inertial and magnetic sensing of human body segment orientation," IEEE Transactions on Neural Systems and Rehabilitation Engineering, vol. 13, no. 3, pp. 395-405, 2005.

[5] A. Tognetti, F. Lorussi, G. Mura, N. Carbonaro, M. Pacelli, R. Paradiso, and D. Rossi, "New generation of wearable goniometers for motion capture systems," Journal of NeuroEngineering and Rehabilitation, vol. 11, 2014.

[6] G. Dalle Mura, F. Lorussi, A. Tognetti, G. Anania, N. Carbonaro, M. Pacelli, R. Paradiso, and D. De Rossi, "Piezoresistive goniometer network for sensing gloves," in XIII Mediterranean Conference on Medical and Biological Engineering and Computing 2013. Springer, 2014, pp. 1547-1550.

[7] N. Carbonaro, G. Dalle Mura, F. Lorussi, R. Paradiso, D. De Rossi, and A. Tognetti, "Exploiting wearable goniometer technology for motion sensing gloves," IEEE Journal of Biomedical and Health Informatics, 2014.

[8] L. Caldani, C. Mancuso, and R. Paradiso, "E-textile platform for movement disorder treatment," in Converging Clinical and Engineering Research on Neurorehabilitation. Springer, 2013, pp. 1049-1053.

[9] H. J. Luinge, P. H. Veltink, and C. T. Baten, "Ambulatory measurement of arm orientation," Journal of biomechanics, vol. 40, no. 1, pp. 78-85, 2007.

[10] R. Bartalesi, F. Lorussi, D. De Rossi, M. Tesconi, and A. Tognetti, "Wearable monitoring of lumbar spine curvature by inertial and etextile sensory fusion," in Engineering in Medicine and Biology Society (EMBC), 2010 Annual International Conference of the IEEE. IEEE, 2010, pp. 6373-6376.

[11] T. M. Sukal, M. D. Ellis, and J. P. Dewald, "Shoulder abductioninduced reductions in reaching work area following hemiparetic stroke: neuroscientific implications," Experimental Brain Research, vol. 183, no. 2, pp. 215-223, 2007.

[12] P. H. Veltink, H. J. Bussmann, W. De Vries, W. L. Martens, and R. C. Van Lummel, "Detection of static and dynamic activities using uniaxial accelerometers," IEEE Transactions on Rehabilitation Engineering, vol. 4, no. 4, pp. 375-385, 1996.

[13] H. M. Schepers, E. H. van Asseldonk, J. H. Buurke, and P. H. Veltink, "Ambulatory estimation of center of mass displacement during walking," IEEE Transactions on Biomedical Engineering, vol. 56, no. 4, pp. 1189-1195, 2009.

[14] "www.biometricsltd.com/gonio.htm."

[15] F. Lorussi, A. Tognetti, N. Carbonaro, G. Anania, and D. De Rossi, "Enhancing the performance of upper limb gesture reconstruction through sensory fusion," in Engineering in Medicine and Biology Society, EMBC, 2011 Annual International Conference of the IEEE. IEEE, 2011, pp. 3496-3499. 